\title{
Comparação entre técnicas de FES na musculatura espástica após AVC: Estudo piloto
}

\author{
Comparison between FES techniques in spastic musculature \\ after stroke: pilot study
}

\section{Comparación entre las técnicas de FES en la musculatura espástica después de un accidente cerebrovascular: estudio piloto}

\author{
Anne Karoline Barros Brasil ${ }^{1}$, Larissa Mielly da Silva Gomes ${ }^{1}$, \\ Mylena Golçalves Fonseca ${ }^{1}$, Carolina Kosour ${ }^{2}$, \\ Adriana Teresa Silva Santos ${ }^{2}$, Luciana Maria dos Reis ${ }^{2}$
}

\begin{abstract}
1.Departamento do curso de Fisioterapia da Universidade Federal de Alfenas, Instituto de Ciências da Motricidade, Alfenas-MG, Brasil.

2.Doutora, Docente do Curso de Fisioterapia da Universidade Federal de Alfenas, Departamento do curso de Fisioterapia da Universidade Federal de Alfenas, Instituto de Ciências da Motricidade, Alfenas-MG, Brasil.
\end{abstract}

\section{Resumo}

Introdução. Entre os diversos recursos terapêuticos utilizados para a reabilitação das sequelas resultantes do acidente vascular cerebral (AVC), destaca-se a estimulação elétrica funcional (FES). Entretanto, pouco se sabe sobre sua aplicação na musculatura espástica, destacando a importância de se contrastar diferentes formas de utilização. Objetivo. Contrastar os métodos de aplicação da FES nos músculos agonista e antagonista em membro superior espástico. Método. Este estudo foi composto por 6 participantes, que receberam sequencialmente as intervenções placebo, FES em bíceps braquial espástico e FES em tríceps braquial, em duas fases de tratamento, imediato e tardio. As avaliações foram realizadas por meio da Eletromiografia, Escala de Ashworth Modificada e Avaliação Fugl Meyer. Resultados. Em ambos os estágios, imediato e tardio, não foram encontrados resultados significativos nas avaliações pré- e pós-tratamento de cada intervenção, nem pós-tratamento na comparação entre intervenções. Conclusão. Não houve diferença entre as aplicações da FES nos músculos agonista e antagonista em membro superior espástico.

Unitermos. Acidente vascular cerebral; Eletroterapia; Musculatura espástica

\begin{abstract}
Introduction. Among the various therapeutic resources used for the rehabilitation of sequel resulting from stroke, functional electrical stimulation (FES) stands out. However, little is known about its application to spastic musculature, highlighting the importance of contrasting different forms of use. Objective. Contrast the methods of application of FES in the agonist and antagonist muscles in spastic upper limbs. Method. This study was composed of 6 participants, who received sequentially the placebo interventions, FES in spastic brachial biceps and FES in brachial triceps, in two phases of treatment, immediate and late. The evaluations were performed by way of Electromyography, Modified Ashworth Scale and Fugl Meyer Assessment. Results. In both immediate and late stages no significant results were found in the pre- and post-treatment evaluations of each intervention, neither after treatment in the comparison between interventions. Conclusion. There was no difference between the applications of FES in the agonist and antagonist muscles in spastic upper limbs.
\end{abstract}

Keywords. Stroke; Electrotherapy; Spastic musculature 


\section{Resumen}

Introducción. Entre los diversos recursos terapéuticos utilizados para la rehabilitación de las secuelas derivadas del ictus, destaca la estimulación eléctrica funcional (FES). Sin embargo, poco se sabe sobre su aplicación a la musculatura espástica, destacando la importancia de contrastar diferentes formas de uso. Objetivo. Contrastar los métodos de aplicación de FES a los músculos agonistas y antagonistas en miembros superiores espásticos. Método. Este estudio estuvo compuesto por 6 participantes, quienes recibieron secuencialmente las intervenciones placebo, FES en bíceps braquial espástico y FES en tríceps braquial, en dos fases de tratamiento, inmediata y tardía. Las evaluaciones se realizaron mediante Electromiografía, Escala de Ashworth Modificada y Evaluación Fugl Meyer. Resultados. Tanto en la etapa inmediata como en la tardía, no se encontraron resultados significativos en las evaluaciones pre- y postratamiento de cada intervención, ni después del tratamiento en la comparación entre intervenciones. Conclusión. No hubo diferencia entre las aplicaciones de FES en los músculos agonistas y antagonistas en miembros superiores espásticos.

Palabras clave. Accidente cerebrovascular; Electroterapia; Musculatura espástica

Trabalho realizado na Universidade Federal de Alfenas, Instituto de Ciências da Motricidade, Alfenas-MG, Brasil.

\section{INTRODUÇÃO}

O Acidente Vascular cerebral (AVC) é oriundo de comprometimentos da irrigação sanguínea em determinada região encefálica, e consequente falta de glicose e oxigênio, que pode gerar danos sensoriais e motores. Essa condição deve-se à causas isquêmicas ou hemorrágicas. O AVC isquêmico é decorrente da carência de suprimento sanguíneo no cérebro, frequentemente dado pela formação de uma placa aterosclerótica ou de um coágulo presente na circulação. O AVC hemorrágico é determinado por ruptura de um vaso sanguíneo e consequente extravasamento do sangue ${ }^{1}$.

A principal sequela neurológica de um AVC é a hemiparesia, caracterizada por paralisia em um hemicorpo 
com consequências e comprometimentos de acordo com a área atingida e que leva a limitações funcionais para o hemicorpo contralateral ao hemisfério lesado. Inicialmente as hemiplegias são dadas por um estado de hipotonia muscular, seguido da instalação de um quadro hipertônico espástico. A espasticidade é clinicamente caracterizada por padrão flexor de membros superiores, com rotação interna e adução do ombro, flexão do cotovelo, pronação e flexão de punho e dedos, e extensão dos membros inferiores, com extensão e rotação interna do quadril, extensão do joelho, flexão plantar e inversão do pé2.

De acordo com o grau de acometimento, o AVC pode ser altamente incapacitante em relação às funções físicas, mentais e cognitivas, o que certamente gera impacto nas atividades de vida diária, restrições na participação social e, consequentemente, piora na qualidade de vida ${ }^{3}$.

Entre os diversos recursos terapêuticos utilizados para a reabilitação de sujeitos com sequelas resultantes do AVC destaca-se a estimulação elétrica funcional (FES). A FES consiste em uma corrente elétrica capaz de estimular conjuntamente o córtex sensorial e motor, promovendo a contração muscular. O controle da espasticidade com a FES pode ser adquirido a partir de dois mecanismos de contração. A estimulação muscular antagonista é conhecida por ter um efeito inibitório no músculo agonista, chamada inibição recíproca. E a estimulação muscular agonista, um método baseado na inibição autógena como via inibidora para o 
próprio agonista, através de um ciclo de feedback negativo para o motoneurônio- $a^{4-7}$.

O objetivo do estudo foi avaliar os efeitos imediatos e tradios da Estimulação Elétrica Funcional (FES), na musculatura espástica de membro superior de sujeitos após Acidente Vascular Cerebral (AVC), bem como comparar a metodologia de estimulação em músculos agonista e antagonista.

\section{MÉTODO}

Trata-se de um estudo piloto controlado sequencial, realizado na Universidade Federal de Alfenas, MG. Foram incluídos no estudo sujeitos que apresentam diagnóstico clínico de AVC, sequelas de hemiparesia direita ou esquerda, tempo de lesão agudo ou crônico, de ambos os sexos, entre 30 e 70 anos de idade, independente de etnia, raça e nível sócio-econômico-cultural, classificados com comprometimento moderado na Escala Fugl Meyer ${ }^{8}$. Foram excluídos sujeitos que apresentavam outro tipo de lesão neurológica associada, que faziam uso de medicamento miorelaxante e que apresentavam espasticidade avaliada em grau 5 pela escala Ashworth Modificada9.

O estudo contou inicialmente com 8 sujeitos, selecionados por conveniência na clínica de Fisioterapia da Universidade Federal de Alfenas. Destes, um indivíduo foi excluído devido ao rastreio de perdas cognitivas, de acordo com o Mini Exame do Estado Mental (MEEM) ${ }^{10}$ e, um segundo 
indivíduo, deixou de participar, logo no início das intervenções, por motivos pessoais.

O estudo foi aprovado pelo Comitê de Ética da UNIFAL (CAAE 82912518.6.0000.5142) e seguiu a Resolução 466/12 do Conselho Nacional de Saúde.

\section{Procedimento}

Os pacientes foram submetidos a dois estágios de tratamento. O primeiro com o objetivo de analisar os resultados imediatos, e o segundo com o objetivo de analisar os resultados tardios da FES.

Para o primeiro estágio foi realizada a avaliação dos pacientes, seguida da intervenção placebo associada ao alongamento do músculo bíceps braquial por 15 minutos, sendo realizada reavaliação imediatamente após a intervenção. O mesmo ocorreu com a FES nos músculos bíceps braquial (agonista espástico) e tríceps braquial (antagonista), porém estes não prosseguiram com a associação do alongamento do músculo bíceps braquial (Figura 1).

Após 72 horas, foi iniciado o próximo estágio com objetivo de avaliar resultados tardios. Este começou com a avaliação inicial dos pacientes e prosseguiu com 8 sessões da intervenção placebo associada ao alongamento do músculo bíceps braquial por 15 minutos, organizadas em 2 aplicações semanais, cessando com a avaliação final dos pacientes. O mesmo ocorreu com a FES nos músculos bíceps braquial e tríceps braquial (Figura 2). 
Figura 1. Estágio de tratamento imediato.

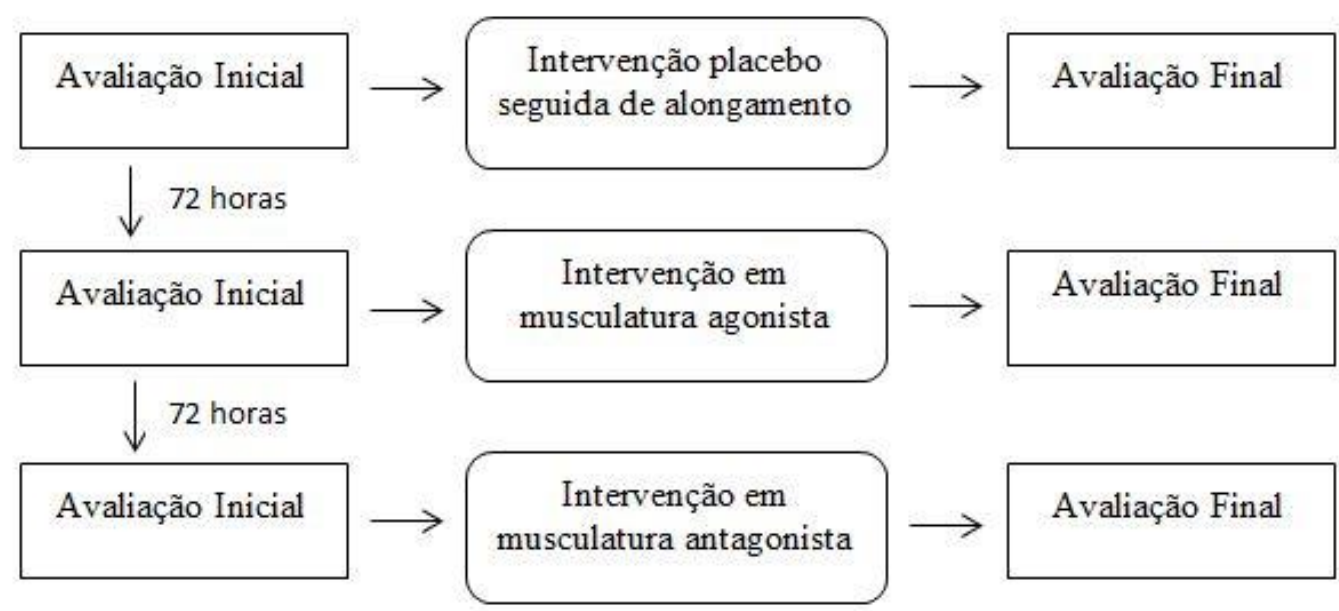

Figura 2. Estágio de Tratamento Tardio.

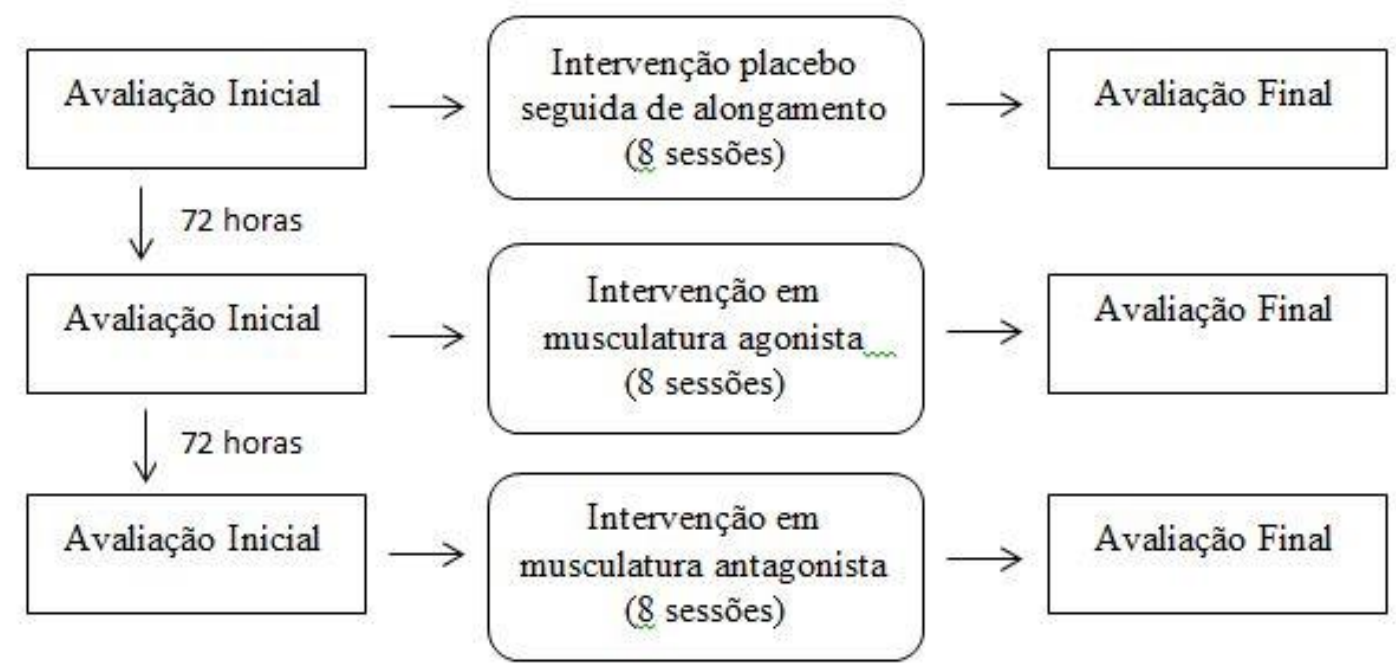

Segundo a literatura, o efeito da FES pode durar até 24 horas, portanto, no presente estudo, foi considerado um intervalo de 72 horas entre as intervenções, como tempo suficientemente longo para garantir que o efeito desapareça e seja dado início à próxima fase ${ }^{2}$. 
Para a intervenção placebo, um eletrodo de referência foi posicionado na origem do músculo bíceps braquial, proximal à parte anterior do braço, adjunto ao acrômio e o outro na região de ponto motor do músculo bíceps braquial espástico. Foi utilizada frequência de $80 \mathrm{~Hz}$, largura de pulso de 300us, com relação ON/OFF de 3:1, sendo o tempo ON de $15 \mathrm{~s}$, subida e descida lentos de 3s, e o tempo OFF de 5s. As sessões tiveram duração de 15 minutos com intensidade $0^{11,12}$.

Para a intervenção no músculo agonista espástico (bíceps braquial), o posicionamento dos eletrodos foi semelhante ao realizado para a intervenção placebo. Foi utilizada frequência de $80 \mathrm{~Hz}$, largura de pulso de 300us, com relação ON/OFF de 3:1, sendo o tempo ON de $15 \mathrm{~s}$, subida e descida lentos de 3s, e o tempo OFF de 5s. As sessões tiveram duração curta, de 10 a 20 contrações ou até ceder a espasticidade ${ }^{13,12}$.

Em relação ao músculo antagonista (tríceps braquial), um eletrodo de referência foi posicionado na origem do músculo tríceps braquial, proximal à parte posterior do braço, lateral a escápula e o outro na região de ponto motor do músculo tríceps braquial, com frequência de $30 \mathrm{~Hz}$, largura de pulso de 250us e relação ON/OFF de 1:3, sendo o tempo ON de $10 \mathrm{~s}$, tempo de subida e descida lentos de $5 \mathrm{~s}$, e tempo OFF de 30s. As sessões tiveram duração de 15 minutos ${ }^{13,14}$. 
Instrumentos de Avaliação

Foi utilizada Eletromiografia (EMG), a partir de um equipamento sistema EMG System do Brasil: Modelo EMG800C. Antes das avaliações serem realizadas, foi feita a tricotomia e a limpeza do local com álcool 70\%, a fim de diminuir a impedância da pele para evitar interferência e permitir melhor aquisição do sinal.

Para a colocação dos eletrodos, foi seguido o protocolo SENIAM ${ }^{15}$ e utilizados eletrodos aderentes monopolares. O músculo avaliado foi o bíceps braquial, em que o eletrodo foi fixado a $1 / 3$ (de baixo para cima) na linha medial entre 0 acrômio e a linha do cotovelo. O eletrodo de referência foi colocado no processo estiloide da ulna do membro contralateral ao lesionado. Na avaliação o indivíduo sentouse em uma cadeira com os pés apoiados no chão, as costas apoiadas e os cotovelos em um ângulo de $90^{\circ}$.

O sinal eletromiográfico foi captado na posição de repouso, a fim de verificar possíveis interferências no local, e posteriormente em contração isométrica voluntária máxima, de 5 segundos, contra uma resistência imposta por uma mesa. Cada coleta foi realizada 3 vezes consecutivas e entre cada coleta com contração, foi dado um intervalo de 2 minutos para haver recuperação do músculo. Foram utilizados comandos verbais para relaxamento e para contração isométrica voluntária máxima.

Além da EMG, foi utilizada a Escala de Ashworth Modificada $(\mathrm{MAS})^{9}$ a fim de mensurar a espasticidade e a 
Fugl Meyer Assessment da extremidade superior ${ }^{16}$ a fim de mensurar o comprometimento motor e sensorial do membro.

\section{Análise Estatística}

Foram considerados para a análise eletromiográfica os dados de valor médio da raiz quadrada (RMS), frequência máxima (PICO) e frequência mediana (FM) das coletas. Além disso, foram analisados os scores da Escala Fugl Meyer (EFM) e da Escala Modificada de Ashwoth (MAS).

Foi utilizado o teste de normalidade de Shapiro Wilk, e devido à natureza não paramétrica dos dados, foi aplicado os testes de Kruskal Wallis. Os dados foram tabulados no Excel Versão 2016 e processados utilizando o software Statistical Package of the Social Sciences (SPSS) Versão 2.0. Em todos os casos, foi adotado o nível de significância de 0,05 .

\section{RESULTADOS}

Foram selecionados 6 sujeitos com diagnóstico de AVC ( 2 homens e 4 mulheres), com idade média de 54,5 anos, prevalência em AVC isquêmico (2:1), com acometimento no hemisfério esquerdo (2:1) e tempo de lesão superior a um ano $(5: 1)$.

Em ambos os estágios, imediato e tardio, não foram encontrados resultados significativos nas avaliações pré- e pós-tratamento de cada intervenção, nem pós-tratamento na comparação entre intervenções (Tabelas 1 e 2). 
Tabela 1. Avaliação pré- e pós-intervenção imediata com FES nas intervenções placebo, musculatura agonista e musculatura antagonista.

\begin{tabular}{|c|c|c|c|c|c|}
\hline$N(7)$ & $\begin{array}{l}\text { EMG } \\
\text { RMS }\end{array}$ & $\begin{array}{l}\text { EMG } \\
\text { PICO }\end{array}$ & $\begin{array}{c}\text { EMG } \\
\text { FM }\end{array}$ & EFM & MAS \\
\hline & $M \pm E P M$ & $M \pm E P M$ & $M \pm E P M$ & $M \pm E P M$ & $M \pm E P M$ \\
\hline $\begin{array}{l}\text { Pré-intervenção } \\
\text { placebo }\end{array}$ & $84,58 \pm 1,93$ & $75,55 \pm 6,06$ & $93,51 \pm 5,64$ & $70,57 \pm 4,13$ & $1,43 \pm 0,30$ \\
\hline $\begin{array}{c}\text { Pós-intervenção } \\
\text { placebo }\end{array}$ & $85,45 \pm 3,14$ & $84,39 \pm 6,16$ & $91,34 \pm 5,10$ & $70,29 \pm 4,07$ & $1,43 \pm 0,30$ \\
\hline $\mathrm{p}^{\mathrm{a}}$ & 1,00 & 0,23 & 0,71 & 0,31 & - \\
\hline $\begin{array}{l}\text { Pré-intervenção } \\
\text { agonista }\end{array}$ & $83,65 \pm 3,86$ & $82,84 \pm 4,11$ & $95,29 \pm 2,91$ & $70,14 \pm 4,02$ & $1,43 \pm 0,30$ \\
\hline $\begin{array}{c}\text { Pós-intervenção } \\
\text { agonista }\end{array}$ & $85,45 \pm 3,14$ & $81,09 \pm 3,26$ & $87,64 \pm 8,86$ & $70,43 \pm 4,00$ & $1,43 \pm 0,30$ \\
\hline $\mathrm{p}^{\mathrm{a}}$ & 0,61 & 0,49 & 1,00 & 0,15 & - \\
\hline $\begin{array}{l}\text { Pré-intervenção } \\
\text { antagonista }\end{array}$ & $77,16 \pm 3,68$ & $73,48 \pm 7,02$ & $91,38 \pm 3,22$ & $70,29 \pm 3,84$ & $1,43 \pm 0,30$ \\
\hline $\begin{array}{c}\text { Pós-intervenção } \\
\text { antagonista }\end{array}$ & $84,15 \pm 4,70$ & $82,10 \pm 3,24$ & $89,94 \pm 3,81$ & $70,29 \pm 3,84$ & $1,43 \pm 0,30$ \\
\hline $\mathrm{p}^{\mathrm{a}}$ & 0,31 & 0,49 & 0,60 & 0,34 & - \\
\hline $\begin{array}{l}\text { Pré-intervenção } \\
\qquad \mathrm{p}^{\mathrm{b}}\end{array}$ & 0,20 & 0,60 & 0,20 & 0,90 & - \\
\hline $\begin{array}{c}\text { Pós-intervenção } \\
\text { p }^{\mathrm{b}}\end{array}$ & 0,90 & 0,30 & 0,70 & 1,00 & - \\
\hline
\end{tabular}

\section{DISCUSSÃO}

O presente estudo avaliou o efeito da FES, em diferentes formas de aplicação, na espasticidade de bíceps braquial após AVC. O protocolo constou de dois estágios de tratamento, imediato e tardio, contendo três grupos em cada estágio: grupo de eletroestimulação com a FES na musculatura agonista (bíceps braquial), grupo de eletroestimulação com a FES na musculatura antagonista (tríceps braquial) e grupo placebo. Em ambos os estágios, imediato e tardio, não foram encontrados resultados significativos nas avaliações pré- e pós-tratamento de cada 
intervenção, nem pós-tratamento na comparação entre intervenções.

Tabela 2. Avaliação pré- e pós-intervenção tardia com FES nas intervenções placebo, musculatura agonista e musculatura antagonista.

\begin{tabular}{|c|c|c|c|c|c|}
\hline $\mathrm{N}(6)$ & $\begin{array}{l}\text { EMG } \\
\text { RMS }\end{array}$ & $\begin{array}{l}\text { EMG } \\
\text { PICO }\end{array}$ & $\begin{array}{c}\text { EMG } \\
\text { FM }\end{array}$ & EFM & MAS \\
\hline & $M \pm E P M$ & $M \pm E P M$ & $M \pm E P M$ & $M \pm E P M$ & $M \pm E P M$ \\
\hline $\begin{array}{l}\text { Pré-intervenção } \\
\text { placebo }\end{array}$ & $190,23 \pm 57,41$ & $118,48 \pm 20,84$ & $100,00 \pm 0,00$ & $75,00 \pm 6,14$ & $1,92 \pm 0,24$ \\
\hline $\begin{array}{l}\text { Pós-intervenção } \\
\text { placebo }\end{array}$ & $111,16 \pm 18,57$ & $123,69 \pm 32,49$ & $94,44 \pm 5,56$ & $77,33 \pm 6,35$ & $1,92 \pm 0,24$ \\
\hline $\mathrm{p}^{\mathrm{a}}$ & 0,05 & 0,60 & 0,32 & 0,07 & 1,00 \\
\hline $\begin{array}{l}\text { Pré-intervenção } \\
\text { agonista }\end{array}$ & $87,08 \pm 8,32$ & $84,52 \pm 11,44$ & $100,00 \pm 0,00$ & $80,33 \pm 5,43$ & $1,92 \pm 0,24$ \\
\hline $\begin{array}{c}\text { Pós-intervenção } \\
\text { agonista }\end{array}$ & $93,35 \pm 4,56$ & $96,14 \pm 10,06$ & $100,00 \pm 0,00$ & $82,17 \pm 5,39$ & $1,75 \pm 0,28$ \\
\hline $\mathrm{p}^{\mathrm{a}}$ & 0,92 & 0,75 & 1,00 & 0,42 & 0,16 \\
\hline $\begin{array}{l}\text { Pré-intervenção } \\
\text { antagonista }\end{array}$ & $96,13 \pm 10,40$ & $104,13 \pm 19,74$ & $108,23 \pm 11,86$ & $82,00 \pm 4,74$ & $1,75 \pm 0,28$ \\
\hline $\begin{array}{l}\text { Pós-intervenção } \\
\text { antagonista }\end{array}$ & $80,09 \pm 4,71$ & $97,73 \pm 20,73$ & $100,00 \pm 0,00$ & $84,83 \pm 4,42$ & $1,75 \pm 0,28$ \\
\hline$p^{a}$ & 0,34 & 0,75 & 0,65 & 0,10 & 1,00 \\
\hline $\begin{array}{l}\text { Pré-intervenção } \\
\qquad \text { Pb }\end{array}$ & 0,04 & 0,40 & 1,00 & 0,41 & 0,72 \\
\hline $\begin{array}{c}\text { Pós-intervenção } \\
\text { Pb }\end{array}$ & 0,09 & 0,48 & 0,37 & 0,47 & 0,72 \\
\hline
\end{tabular}

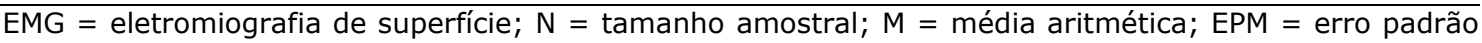
da média; $\mathrm{p}^{\mathrm{a}}=$ significância intra-grupos; $\mathrm{p}^{\mathrm{b}}=$ sugnificância inter-grupos; $\mathrm{RMS}=$ valor médio da raiz quadrada; PICO = frequência máxima; FM = frequência mediana; EFM = Escala Fugl Meyer; MAS = Escala Modificada de Ashwoth.

Uma revisão sistemática e meta-análise ${ }^{17}$, analisou a eficácia da FES em membros superiores após AVC nas atividades de vida de diária e função motora. Dentre os estudos selecionados, vinte foram incluídos na revisão. O tempo médio desde o AVC, em 15 dos estudos selecionados, foi de 2,87 anos com variação considerável: cinco estudos relatando tempos médios inferiores a 2 meses, cinco relatando tempos médios entre 1 e 3 anos e 
seis relatando tempos médios ao longo de 3 anos. Nos estudos, em que a FES foi iniciada em tempo médio pós-AVC inferior a 2 meses, observou-se um benefício significante da FES e heterogeneidade estatística moderada. Em contraste, nos estudos em que a FES foi iniciada com tempo médio maior que 1 ano após o AVC, nenhuma melhora significante foi observada.

Em outro aspecto, os estudos analisados não utilizaram os mesmos parâmetros no protocolo de estimulação com a FES, e os instrumentos de avalição também não foram padronizados entre eles. Essa variabilidade nos parâmetros da FES pode influenciar os resultados desta revisão e ser um fator que contribui para a heterogeneidade nas análises. Desta forma, os autores concluem que possivelmente a FES seja benéfica quando aplicada usando parâmetros de estimulação específicos ou quando aplicada a uma população específica, sugerindo pesquisas futuras para identificar os parâmetros ideais da FES e padronizar os tratamentos realizados ${ }^{17}$.

Diante do exposto na revisão sistemática ${ }^{17}$, uma possível justificativa para os resultados do presente estudo, seja a não seleção dos participantes por tempo de acometimento do AVC, sendo a proporção dos participantes em estágio crônico foi superior aos que estavam em estágio agudo (5:1).

O uso da FES e a terapia de espelho foram analisadas por meio da eletromiografia, em 13 pacientes com espasticidade em membro superior após AVC agudo, durante 
oito semanas, associado também a outras terapias, como fisioterapia convencional e terapia ocupacional ${ }^{18}$. Os músculos alvo de estimulação foram o extensor radial do carpo e o extensor comum dos dedos, com modulação de frequência em $50 \mathrm{~Hz}$, largura de pulso de 200us, tempo ON de 10 s e tempo de OFF de 20s. Em contradição ao presente estudo, houve resultado significante na melhora do comprometimento motor e sensorial pela EFM ${ }^{18}$. No entanto, o estudo contou com tamanho amostral superior ao do presente estudo, e todos os participantes estavam em período agudo após lesão.

O efeito da FES no músculo agonista e antagonista de sujeitos com hemiparesia espástica decorrente de AVC, foi comparada a partir de uma revisão sistemática ${ }^{2}$. Os artigos analisados sugerem que tanto a FES em músculos agonistas quanto em antagonistas são métodos de tratamento úteis na reabilitação de sujeitos hemiparéticos espásticos, porém há escassez literária quanto a ensaios clínicos comparativos de tais métodos, sendo este um campo de estudo importante para a efetividade da intervenção fisioterapêutica visando à reabilitação dos pacientes hemiparéticos.

Para mais, este estudo piloto poderá contribuir para futuras pesquisas na área, uma vez que, embora a FES seja considerada uma intervenção clínica convencional, seus parâmetros e formas de utilização necessitam de maiores esclarecimentos. Desta forma, sugere-se a realização de novos estudos com maior tamanho amostral e técnicas aplicadas em grupos independentes. 


\section{CONCLUSÃO}

No presente estudo, não foram encontrados resultados significativos nas avaliações pré- e pós-tratamento de cada intervenção, nem pós-tratamento na comparação entre intervenções em ambos os estágios, imediato e tardio. Portanto, não houve diferença entre as aplicações da FES nos músculos agonista e antagonista do membro superior espástico.

\section{REFERÊNCIAS}

1.Silva RF, Lima RD. A importância da fisioterapia precoce na recuperação do controle motor após AVC (TCC). Bragança Paulista: Universidade São Francisco, 2016; 34p. http://lyceumonline.usf.edu.br/salavirtual/documentos/2738.pdf

2.Souza DQS, Mendes IS, Borges ACL, Fresitas STT, Lima FPS, Lima $\mathrm{MO}$, et al. Efeito da estimulação elétrica neuromuscular (EENM) no músculo agonista e antagonista de sujeitos com hemiplegia espástica decorrente da disfunção vascular encefálica: Revisão sistemática. Rev Univap 2011;17:58-67. http://doi.org/10.18066/revunivap.v17i30.41 3.Araújo APS, Silva PCF, Moreira RCPS, Bonilha SF. Prevalência dos fatores de risco em pacientes com acidente vascular encefálico atendidos no setor de neurologia da clínica de fisioterapia da UNIPAR, campus sede. Arq Cienc Saúde Unipar 2008;12:35-42. https://revistas.unipar.br/index.php/saude/article/viewFile/2226/183 $\underline{8}$

4.Lima-Junior FAV, Silva WHS, Costa FA. Impacto do acidente vascular cerebral no cotidiano de cuidadores familiares. Est Interdisc Envel 2012;17:251-64. https://doi.org/10.22456/2316-2171.22871

5.Salm A, Veltink PH, IJzerman MJ, Groothuis-Oudshoorn KC, Nene AV, Hermens $\mathrm{HJ}$. Comparison of electric stimulation methods for reduction of tríceps surae spasticity in spinal cord injury. Arch Phys Med Rehabil 2006;87:222-8. https://doi.org/10.1016/j.apmr.2005.09.024

6. Oshiro SH, Oliveira CL, Bim ACS, Oliveira GSR, Riberto M. Functional electrical stimulation optimized in patients with hemiparesis due to cerebrovascular disease. Acta Fisiatr 2012;19:46-9. http://doi.org/10.5935/0104-7795.20120010

7.Santos RCM, Carvalhais OC, Paz CCSC, Criollo CJT. Uso da estimulação elétrica functional pós acidente vascular cerebral: revisão sistemática. Rev Neurocienc 2015;23:103-15. http://doi.org/10.4181/rnc.2015.23.01.1008.13p 
8. Maki T, Quagliato EMAB, Cacho EWA, Paz LPS, Nascimento NH, Inoue MMEA, et al. Estudo de confiabilidade da aplicação da escala de FuglMeyer no Brasil. Rev Bras Fisiot 2006;10:177-83. http://doi.org/10.1590/s1413-35552006000200007

9. Corrêa ACS, Silva JDS, Silva LVC, Oliveira DA, Cabral ED. Crioterapia e cinesioterapia no membro superior espástico no acidente vascular cerebral. Fisioter Mov 2010;23:555-63. https://doi.org/10.1590/S0103-51502010000400006

10. Melo DM, Barbosa AJG. O uso do Mini-Exame do Estado Mental em pesquisas com idosos no Brasil: uma revisão sistemática. Cienc Saúde Col 2015;20:3865-76.

http://doi.org/10.1590/1413-

812320152012.06032015

11.Ambrosini E, Ferrante S, Pedrocchi A, Ferrigno G, Molteni F. Cycling Induced by Electrical Stimulation Improves Motor Recovery in Postacute Hemiparetic Patients. Stroke 2011;42:1068-73. http://doi.org/10.1161/strokeaha.110.599068

12.Silva DD, Borges ACL, Lima MO, Lima FPS, Freitas STT, Nogueira DV, et al. Resistência ao movimento e atividade eletromiográfica dos músculos flexores e extensores de cotovelo em pacientes hemiparéticos espásticos submetidos à crioterapia e estimulação elétrica neuromuscular. Rev Bras Eng Biomed 2012;28:248-60. http://doi.org/10.4322/rbeb.2012.025

13.Scheker LR, Chesher SP, Ramirez S. Neuromuscular electrical stimulation and dynamic bracing as a treatment for upper-extremity spasticity in children with cerebral pals. J Hand Surg 1999;24:226-32. https://doi.org/10.1054/JHSB.1998.0002

14.Felice TD, Ishisuka ROR, Amarilha JD. Eletroestimulação e Crioterapia para espasticidade em pacientes acometidos por Acidente Vascular Encefálico. Rev Neurocienc 2011;19:77-84. https://doi.org/10.34024/rnc.2011.v19.8415

15.SENIAM Project. Surface ElectroMyoGraphy for the Non-Invasive Assessment of Muscles (endereço na internet). (acessado em 21/03/2018). Disponível em: http://www.seniam.org

16.Padovani C, Pires CVG, Ferreira FPC, Borin G, Filippo TRM, Imamura $M$, et al. Aplicação das escalas Fugl-Meyer Assessment (FMA) e Wolf Motor Function Test (WMFT) na recuperação funcional do membro superior em pacientes pós-acidente vascular encefálico crônico: revisão de literatura. Acta Fisiátr 2013;20:42-9. http://doi.org/10.5935/0104-7795.20130008

17.Eraifej J, Clark W, France B, Desando S, Moore D. Effectiveness of upper limb functional electrical stimulation after stroke for the improvement of activities of daily living and motor function: a systematic review and meta-analysis. Syst Rev 2017;6:40. http://doi.org/10.1186/s13643-017-0435-5

18. Kosuke K, Koki I, Yuta M, Kentaro T, Shigeru M, Koji S. Feasibility study of a combined treatment of electromyography-triggered neuromuscular stimulation and mirror therapy in stroke patients: A randomized crossover trial. J Neurorehabil 2014;34:235-44. http://doi.org/10.3233/NRE-131038 